\title{
像增强器的迭代性能及其评价标准
}

\author{
潘京生 ${ }^{1,2}$ \\ （1. 微光夜视技术重点实验室, 陕西 西安 710065；2. 北方夜视技术股份有限公司, 江苏 南京 211102）
}

\begin{abstract}
摘要: 超二代像增强器与三代像增强器长期保持着并行发展的态势, 但迭代性能划定和命名之争 也贯穿其发展历程。随着多碱光电阴极的光谱拓展取得实质性突破, 同时无膜微通道板在一些 GaAs 光阴极像增强器中也成为可能, 像增强器的 “代” 已彻底失去意义, 可对各自所能达到的 实际使用性能仍然缺之一个全面准确的评判。通过回顾像增强器的发展历程及其标志性的迭代性 能突破, 和随之多次引发的迭代性能划定和命名之争, 进一步说明标准规范在准确评价像增强器 的迭代性能特征及其实际使用性能方面存在不足, 同时, 数字化可集成的夜视技术是未来的发展 趋势, 数字像增强相机也已成为像增强技术的一种新的产品形态, 像增强器的标准更新及其性能 参数与实际使用性能对应关系的研究已经显得很有必要。
\end{abstract}

关键词：夜视；像增强器；信噪比；分辨力；可靠性

中图分类号: TN223 文献标识码: A 文章编号：1001-8891(2020)06-0509-10

\section{Image Intensifier Upgraded Performance and Evaluation Standard}

\author{
PAN Jingsheng ${ }^{1,2}$ \\ (1. Science and Technology on Low-Light-Level Night Vision Laboratory, Xi'an 710065, China; \\ 2. North Night Vision Technology Corp. Ltd., Nanjing 211102, China)
}

\begin{abstract}
The Gen 2 image intensifier is keeping a parallel technology development status with the Gen 3 image intensifier and the debate over the generation division and performance upgrade is also accompanying their development. Over time, as substantial breakthroughs for multi-alkali photocathode in the extended spectral range occur, as well as the development of filmless micro-channel plates(MCP) in some GaAs photocathodes image intensifiers, the term "Generation" for image intensifiers has arguably lost meaning. However, there is still lack of accurate comparison assessments for the filed performance that both generations have achieved. Though the existing military specification cannot fully reflect the upgraded image intensifier characteristic and its actual field performance, standards updates as well as investigations of the link between these characteristic parameters and the actual field performance are necessary.
\end{abstract}

Key words: night vision, image intensifier, signal to noise ratio, resolution, reliability

\section{0 引言}

夜视是为获得夜间战术优势采用的被动式辅 助视觉, 通常采用一个宽视场角的 $1: 1$ 倍率的光学 系统, 因而也称为夜视镜或夜视仪, 依赖于月光及 星光包括太阳光在夜天空的直接和非直接的微弱 照明, 以及由外大气层不同组份所释放的微弱辐射 的存在, 借助于一种称为像增强器的真空电子光学
器件, 将微弱影像增强到目视可观察的亮度级别。 像增强器也称为像管, 由物镜收集到的那些由景物 反射的可见光和近红外光子, 在这里经阴极输入窗 内的光阴极转换成光电子后逸出真空, 经过放大, 在阳极输出窗荧光屏上转换为适合于目视观察亮 度级别且与观察景物高度空间匹配的单色影像, 然 后再通过目镜光学放大直视观察。

像增强器这一传统技术曾几度被认定已经过 
时且将逐渐消亡, 但其可满足于无月星光微弱照度 下的快速成像, 以及紧凑、轻便和低功耗的可穿戴 装备集成能力, 至今仍不能为其他技术所替代和超 越。像增强器同时还存在着两种不同技术路线的竞 争, 多碱光阴极超二代像增强器已发展成为与三代 像增强器并行的技术, 但迭代性能划定和命名之争 也贯穿于其发展历程 ${ }^{[1-5]}$ 。随着多碱光电阴极的光 谱拓展取得实质性突破, 同时无膜微通道板在一些 GaAs 光阴极像增强器中也成为可能, 像增强器的 “代” 已彻底失去意义, 但对各自所能达到的实际 使用性能仍然缺乏一个全面准确的评判。本文通过 回顾像增强器的发展历程及其迭代性能的里程碑 标志, 和随之引发的多次迭代性能划定及命名之 争, 来说明现有军标规范在准确评价像增强器的迭 代性能及其实际使用性能方面存在不足, 像增强器 的标准更新及其特征参数与实际使用性能对应关 系的研究必要而迫切。

\section{1 像增强器的发展历程及其迭代性能之争}

要构筑一个可用于无月星光夜晚的像增强系 统, 系统增益满足人眼视雉细胞视觉的光适应状 态, 需要 $3000 \mathrm{~cd} \cdot \mathrm{m}^{-2} \cdot 1 \mathrm{x}^{-1}$ 以上的亮度增益。一代 像增强器, 采用聚焦雉电子透镜聚焦及电子加速的 能量增强方式, 不足以达到这个增益级别, 需采用 级联方式, 不能满足军事夜视装备的要求。微通道 板 (microchannel plate, MCP) 的成功引入使像增强 原理从电子加速转为电子倍增, 随之演变而来的双 近贴管结构, 使紧凑、轻便和低功耗的可穿戴夜视 装置成为可能, 奠定了现代像增强器的技术基础。

$\mathrm{MCP}$ 是一个由规则排列的还原铅硅酸盐玻璃 微细通道 (孔径 5 10 $\mu \mathrm{m}$ ) 构成的玻璃薄片, 上下 两端面镀有金属电极。当施加一个高压, 每个通道 即为一个电子倍增通道, 由此构成一个通道式电子 倍增器阵列。早期二代管仍为聚焦雉锐聚焦的倒像 管, 但很快就被平行电场聚焦的近贴管所取代, 如 图 1(a)所示, 来自光阴极的光电子, 在这里经由一 个 $200 \mathrm{~V}$ 电压近百微米间距的近贴聚焦, 空间对应 的进入 MCP 通道, 在一个约 $800 \mathrm{~V}$ 的 MCP 电压下, 获得近 $10^{3}$ 的电子增益, 再经一个 $6 \mathrm{kV}$ 约 $500 \mu \mathrm{m}$ 的近贴聚焦电场的加速, 撞击沉积在光纤输出窗背 面的苂光粉, 苂光粉表面覆以铝膜, 以提高发光效 率, 并减少电荷残留, 获得一个约 $10^{2}$ 的光子/电子 转换效率, 同时结合光纤倒像器直接图像反转, 转 换为高亮度的可见光图像。苂光粉发光光谱对应于 人眼过渡视觉灵敏范围, 亮度增益取决于 MCP 增 益, 苂光粉发光效率和光阴极探测效率。
像增强器电源采用半开合环绕式构造与像管 管壳灌装成一体, 由两个甚至一个 “AA” 电池供 电维持 $40 \mathrm{~h}$ 以上的连续工作, 并在高光照级别环境 使用时为像增强器提供的自动亮度控制和亮光源 保护功能。前者在输入光级别相应于约 $1 / 4$ 月的环 境照度以上启动, 自动调整 $\mathrm{MCP}$ 电压, 确保荧光 屏输出亮度不超过规定的优化范围, 即像增强器的 最大亮度阈值, 也称为饱和度。后者可在遭遇超量 亮度光源辐照时, 自动降低光阴极与 $\mathrm{MCP}$ 间的前 近贴聚焦电压, 避免引发图像浮散乃至像管损坏, 但也导致了分辨力的下降。

更高的亮度增益并不意味着具备在黑暗中看 得更远更清楚的能力, 像增强器的性能更多的取决 于信噪比和调制传递函数 (modulation transfer function, MTF) 相关或派生的参数, 信噪比为像 增强器输出到眼睛的光信号与眼睛感知到的噪声 的比, 与光阴极探测效率的平方根成正比。MTF 可量化输出图像相对于其输入图像的对比度减退 程度，极限分辨力相应于 MTF 在 3\% 5\%范围所 对应的空间频率, 通常也称为分辨力。业内广泛采 用由弱光指标信噪比和强光指标极限分辨力组合 成的品质因数 (figure of merit, FOM) 来评价像增 强器的性能。

像增强器按光电阴极有效直径分类, $18 \mathrm{~mm}$ 管 是夜视仪的典型管型。军标规范采用 $2856 \mathrm{~K}$ 铇丝 白炽灯为标准光源, 以 $108 \times 10^{-6} 1 \mathrm{x}$ 作为输入照度 来表征信噪比, 这个照度相当于无月星光时的地面 照度。以每单位输入光通量的光阴极输出电流来表 征光阴极灵敏度, 也称为白光灵敏度。亮度增益是 荧光屏输出亮度 $\left(\mathrm{fL}\right.$ 或 $\left.\mathrm{cd} / \mathrm{m}^{2}\right)$ 与入射在光电阴极 上的输入照度 ( $\mathrm{fc}$ 或 $1 \mathrm{x})$ 之比 ( $\mathrm{fL} / \mathrm{fc}$ 除以 $\pi$ 转换为 $\mathrm{cd} \cdot \mathrm{m}^{-2} \cdot \mathrm{x}^{-1}$ 的 SI 单位)。可靠性是在给定时间范围 不发生失效的概率，也称为平均失效前时间 (mean time to failure, MTTF)。像增强器的性能随使用时 间的累积而逐渐退化, 失效判据为关键性能指标降 低到规定阈值之下, 如亮度增益、等效背景照度 (equivalent background illumination, EBI)、信噪比、 成像质量、亮度均匀性等, 信噪比和亮度增益的衰 减是主要的失效原因。信噪比和亮度增益的失效阈 值为额定起始值的 $50 \%$ 。

二代管的 S-25 光电阴极与一代管的 S-20 同为 无定型的多碱组份, 采用 $\mathrm{Sb}$ 与 $\mathrm{Na} 、 \mathrm{~K} 、 \mathrm{Cs}$ 交替蒸 镀并反应的制造工艺, 当时 S-20 的灵敏度约 180 $\mu \mathrm{A} / \mathrm{lm}$, 响应范围从紫外覆盖到 $850 \mathrm{~nm}$ 。S-25 增加 了发射层厚度, 减小蓝响应, 拓展了红响应, 响应 范围拓展到 $900 \mathrm{~nm}$, 二代管的光阴极灵敏度提高到 
$350 \mu \mathrm{A} / \mathrm{lm}$, 二代加的光阴极灵敏度则提高到 400 $450 \mu \mathrm{A} / \mathrm{lm}$, 信噪比达到 12 , 分辨力 $30 \mathrm{lp} / \mathrm{mm}$, 亮 度增益达到 $3000 \sim 6000 \mathrm{~cd} \cdot \mathrm{m}^{-2} \cdot 1 \mathrm{x}^{-1}$, 可靠性 MTTF 为 $2500 \sim 4000 \mathrm{~h}$ 。

二代像增强器奠定像增强器在军用夜视装备 中的技术基础。像增强器原理与结构至今未发生大 的改变, 之后像增强技术的发展都是围绕着光阴极 灵敏度的提升和响应范围拓展, 以及 $\mathrm{MCP}$ 功效的 改善来展开, 包括自动选通。而随后的三代像增强 器与迭代进步的超二代像增强器之间的界限变得 越来越模糊不清, 迭代划定和命名之争也贯穿像增 强器的发展始终。

\section{1 三代像增强器与超二代像增强器}

三代像增强器出现在 20 世纪 80 年代, 与二代 像增强器原理结构基本相同, 如图 1 所示, 但有两 个显著特征, 负电子亲和势 (negative electron affinity, NEA) 的 GaAs 光电阴极和 MCP 的离子阻 挡膜。三代管的光电阴极采用 $\mathrm{Al}_{1-x} \mathrm{Ga}_{x} \mathrm{As} / \mathrm{GaAs}$ 的 异质结构, 以热膨胀匹配的 $\mathrm{Al}_{1-x} \mathrm{Ga}_{x} \mathrm{As}$ 窗口层与 7056 玻璃输入窗热压粘接, 通过 $\mathrm{Cs}$ : $\mathrm{O}$ 激活, 在原 子级清洁的 GaAs 表面生成的 GaAs-O-Cs:Cs-O-Cs 双偶极层, 形成 NEA 的光阴极, 可使导带底部的 光电子自由逸出。但 MCP 工作状态下的离子反馈, 撞击光阴极将导致 NEA 功效的迅速衰竭, 为此需 要在 $\mathrm{MCP}$ 输入面上制作一层 $\mathrm{Al}_{2} \mathrm{O}_{3}$ 的薄介质膜, 以阻止反馈离子从通道逸出, 并采用了双铟封的结 构和 $10^{-9} \mathrm{~Pa}$ 超高真空封装制程。但为优化离子阻 挡膜的电子透射特性, 三代管增大了 $\mathrm{MCP}$ 与光阴 极的间距和场强, 导致 halo 尺寸变大。halo 是明亮 的光源被一个比正常的点扩散函数要大得多的晕 轮所包围的现象, 是来自光阴极的光电子在 MCP 输入表面的散射和弹性反射的结果, 这个反弹距离 可达到光阴极与 $\mathrm{MCP}$ 的近贴间距的两倍。

$\mathrm{GaAs}$ 光阴极具有极好的灵敏度, 响应范围上限 为 $\mathrm{GaAs}$ 带隙对应的 $900 \mathrm{~nm}$ (室温), 虽然 $\mathrm{Al}_{1^{-}{ }_{x} \mathrm{Ga}_{x} \mathrm{As}}$ 的带隙限定了对 $600 \mathrm{~nm}$ 以下的响应度, 但相比于 S-25 光电阴极, 灵敏度有显著提升, 并与星光的光 谱分布也有更好的匹配。而无月星光夜天空在 800 $\mathrm{nm}$ 以上近红外波段的丰富辐射, 特别是该波段自 然和人工物体反射率存在的显著差异, 使 $\mathrm{GaAs}$ 光 阴极的 $800 \mathrm{~nm}$ 以上近红外响应优势得以充分体现, 如图 2 和图 3 所示, 可在无月星光的夜晚提供更丰 富的场景信息和增强的场景对比度, 有助于夜间地 形特征识别 ${ }^{[5-7]}$, 三代像增强器成为像增强技术的 又一里程碑标志。

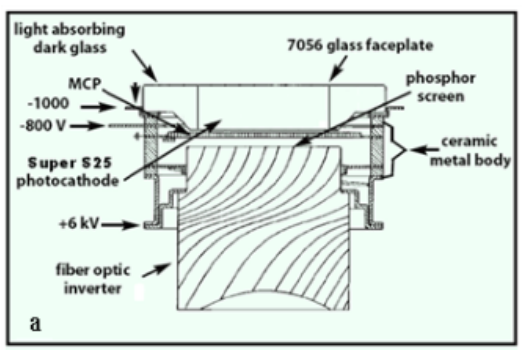

(a) Gen 2 tube

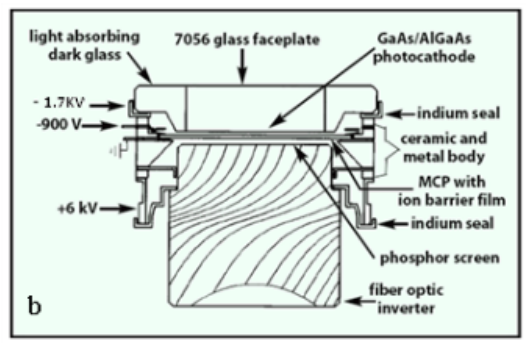

(b) Gen 3 tube

图 1 二代管和三代管的结构差别

Fig.1 Difference between Gen 2 tube and Gen 3 tube

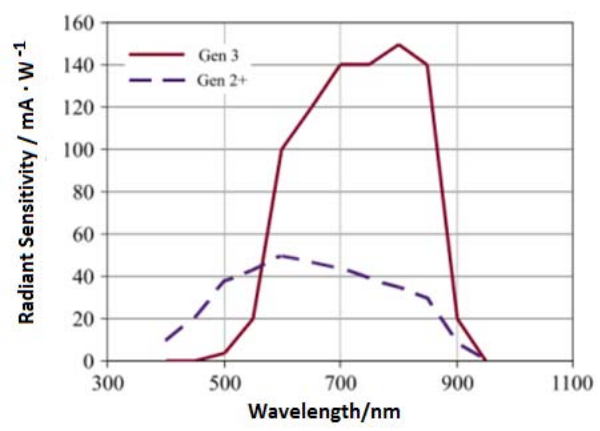

图 2 早期三代与二代加光阴极辐射灵敏度曲线

Fig. 2 Radiant sensitivity of GaAs photocathode and S-25

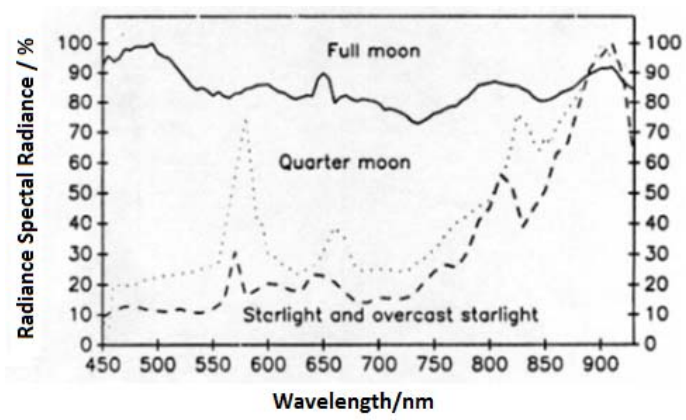

图 3 不同夜天空的 $450 \sim 900 \mu \mathrm{m}$ 波段的辐射

Fig. 3 Detailed night sky radiant in the $450-900 \mu \mathrm{m}$ band

早期三代管光阴极灵敏度接近 $1000 \mu \mathrm{A} / 1 \mathrm{~m}$, 暗 电流低至 $10^{-16} \mathrm{~A} / \mathrm{cm}^{2}$, 亮度增益达到 $50000 / \pi \sim$ $80000 / \pi \mathrm{cd} \cdot \mathrm{m}^{-2} \cdot \mathrm{xx}^{-1}$ ，可靠性达到 $7500 \sim 10000 \mathrm{~h}$ 。 1982 年美国陆军开启 Omnibus 夜视装备采购计划, 为大规模军用夜视装备奠定了基础, 像增强器性能 随之逐步提高。Omni VII 前历次 Omnibus 采购都以 FOM 划定最低性能要求。Omin I 的信噪比是 16.2 , 
极限分辨率为 $36 \mathrm{lp} / \mathrm{mm}$ 。而从 1990 年 Omni III 到 1996 年 Omni IV，阴极灵敏度从 $1350 \mu \mathrm{A} / \mathrm{lm}$ 提高 到 $1800 \mu \mathrm{A} / \mathrm{lm}$, 信噪比从 19 提高到 24 , 分辨力从 $56 \mathrm{lp} / \mathrm{mm}$ 提高到 $64 \mathrm{lp} / \mathrm{mm}^{[3]}$ 。

ITT 于 1995 年发布的 Ultra 管, 采用更小孔径 和更大开口面积比的 $\mathrm{MCP}$, 来弥补三代管在近贴聚 焦上存在的不足。获得次年 Omni IV 大部份采购份 额, 美军标的信噪比失效阈值也提高到了 16 , 可靠 性 MTTF 达到 $10000 \mathrm{~h}$ 。

Photonis 和 DEP 持续致力于多碱光阴极灵敏度 的提高及近红外响应拓展。随着自动控制的多碱光 阴极薄膜生长技术以及像增强组件封装的精细化 工艺推进, S-25 光阴极灵敏度提高到 $500 \mu \mathrm{A} / \mathrm{lm}$ 以 上, 近红外响应拓展到 $900 \mathrm{~nm}$, 多碱光阴极像增强 器性噪比达到 18 , 分辨力 $45 \mathrm{~lm} / \mathrm{mm}$, 工作寿命提 升到 $10000 \mathrm{~h}$, 亮度增益也逐步提高到 6400 11000 $\mathrm{cd} \cdot \mathrm{m}^{-2} \cdot 1 \mathrm{x}^{-1}$ 。 Photonis 和 DEP 于 1995 年将二代加 升级为 Super Gen, 超二代像增强技术渐成雉形。

离子阻挡膜削弱了 $\mathrm{GaAs}$ 光阴极灵敏度的优 势。超二代像增强器的 FOM 达到与三代管相当的 水平。通过引入噪声因子的概念, 可对 MCP 放大 传递过程所导致的光阴极输出信号的信噪比的降 低和损失程度, 在三代管和超二代管这两种状态, 进行比拟:

$$
N_{\mathrm{f}}=\frac{(S / N)_{\text {in }}}{(S / N)_{\text {out }}}=k \frac{\sqrt{S_{k}}}{(S / N)_{\text {out }}}
$$

式中: $N_{\mathrm{f}}$ 为像增强器的噪声因子; $(S / N)_{\text {in }}$ 是光阴极 输入信号的信噪比; $(S / N)_{\text {out }}$ 是像增强器的输出信噪 比; $S_{k}$ 是光阴极灵敏度, $k$ 为对应于标准测试条件 误差的校正系数。苂光屏转换和传递过程的统计涨 落, 以及积分时间导致时间相近事件的重叠增加的 噪声, 可通过一个校正因子 $K$ 的 “噪声平滑” 来去 除。这样 $(S / N)_{\text {out }}$ 仅针对光阴极和 $\mathrm{MCP}, N_{\mathrm{f}}$ 则仅针 对 MCP。标准三代管的噪声因子达到了 2.0, 而超 二代管仅为 1.4 。

美国一直沿用二代管来称呼这种迭代性能进 步的多碱光阴极像增强器。为回避由三代管主导的 迭代划分, Photonis 和 DEP 采用自己的命名方式来 命名此后的性能进步, 如 DEP 的 SHD $-3^{\mathrm{TM}}$ 和 $\mathrm{XD}-4^{\mathrm{TM}}$ ( super high definition 和 eXtended Definition), 以及后来的 XR $-5^{\mathrm{TM}}$ (eXtended Range)。 而国内则用超二代来泛指这些具有迭代性能进步 的多碱光阴极像增强器。但按 Photonis 和 DEP 的 命名体系, SuperGen 和 SHD-3 就高于了超二代, XD-4 和 XR5 已不亚于美国三代。(于是后来的迭
代产品则命名为了 $4 \mathrm{G}$, 这是后话)。

XD4 的光阴极灵敏度突破 $700 \mu \mathrm{A} / \mathrm{lm}$, 信噪比 达到 24 , 分辨力达到 $64 \mathrm{lp} / \mathrm{mm}$, 亮度增益也从早期 的 $8000 \sim 12000 \mathrm{~cd} \cdot \mathrm{m}^{-2} \cdot \mathrm{lx}^{-1}$ 提高到 $10000 \sim 18000$ $\mathrm{cd} \cdot \mathrm{m}^{-2} \cdot 1 \mathrm{x}^{-1}$ 。虽然在亮度增益上低于三代管的水 平, 但 DEP 认为强光级别的输出亮度本身即受电 源预先设定的饱和度的限定, 而微弱光级别更高的 亮度增益无助于噪声的改善, 宣称 XD-4 的性能达 到 Omni IV 三代管的水平, 在 halo 和低频段的 MTF 还更具优势, 还通过引入光谱信噪比的概念, 如图 4 所示, 来说明 XD-4 在海边和沙漠等蓝/绿光丰富 的环境具有优势, 而 Omni IV 三代管在从林等近红 外丰富的环境具有优势 ${ }^{[1]}$ 。但这个光谱信噪比一直 也没有能够建立起相应的计量标准和测试方法。

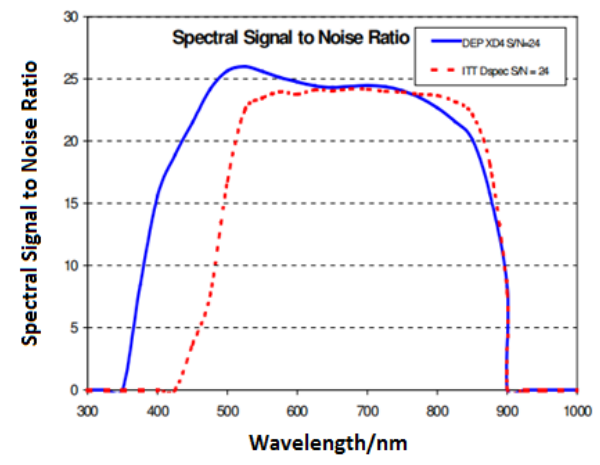

图 $4 \mathrm{DEP}$ 将光电阴极量子效率乘以白光信噪比作为光谱 信噪比对 XD-4 与三代 Omni IV 的对比曲线

Fig.4 Spectral SNR curve based on QE multiplied by SNR which DEP used for comparison of XD-4 and GEN 3 Omni IV

更高的亮度增益 (不高于 $30000 \mathrm{~cd} \cdot \mathrm{m}^{-2} \cdot 1 \mathrm{x}^{-1}$ )

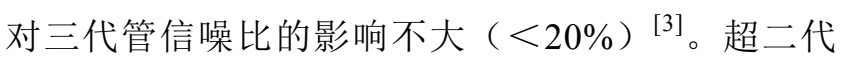
管缺乏离子阻挡膜对离子反馈的有效抑制, 真空度 也相对不足，离子反馈虽不会导致 S-25 光阴极灵 敏度的快速下降, 过高的增益只会增加离子噪声而 无助于图像质量改善, 适度的亮度增益成为超二代 管的一个折衷选择。

\section{2 自动选通与无膜三代、薄膜三代和高性能超二 代}

自动选通电源借助于 $\mathrm{MCP}$ 与光阴极间的聚焦 电场实施的脉冲电压, 并自动切换和调节光阴极选 通开/关及其周期占空比, 平抑高输入光照时光阴极 产生的时间平均光电子流, 消除过量光电子流引发 的图像浮散, 确保在环境亮度发生快速变化时荧光 屏的输出亮度变化低于 $0.17 \mathrm{~cd} / \mathrm{m}^{2}$, 在 $200 \mathrm{~lx}$ 照度 的分辨力下降不超过 $30 \% \sim 40 \%$, 而非选通像增强 器通常下降高达 $80 \%{ }^{[8]}$ 。

自动选通堪称像增强器技术的又一次里程碑 
标志, 无论是对三代还是超二代, 但自动选通却源 于美国的 “四代像增强器” 开发计划。实现无膜 $\mathrm{MCP}$ 的 “四代像增强器”一度被认为是三代之后的 下一个顺理成章的发展目标。1997 年美国陆军 NVESD 召集 Litton 和 ITT 开展无膜 MCP 并发展为 四代像增强器的研究。次年初在陆军 Omni V 竞标 中, Litton 提出的 “自动选通十无膜 MCP” 的 “四 代管” 开发和交付方案, 获得了大部份采购份额。

虽然自动选通可减少 MCP 在高光照期间的工 作时间及相应的反馈离子, 但也相应增加了应用严 酷等级。要去除离子阻挡膜, 需要大幅度的减少 $\mathrm{MCP}$ 工作状态产生的反馈离子数量, 同时改善 GaAs 光阴极耐受反馈离子损伤的能力。Litton 采用 了一种高性能玻璃 $\mathrm{MCP}$, 同时, 采用 Cs:F:O 激活 的 NEA 层以改善表面偶级层的稳定性, 提高 NEA 层对反馈离子的耐抗性。在 1999 年 Litton 为 NVESD 演示的两只 “无膜四代管” 分别达到 32.6 和 35.8 的信噪比 ${ }^{[9-10]}$ 。OMN IV 要求 FOM 不低于 1800 , 信噪比目标值 30 , 最低值 27 。但 Litton 无 膜管交付率极低, 在交付后还发现在高光照和高温 环境使用时性能快速退化。

ITT 调整了技术方案, 减薄离子阻挡膜而非完 全去除, 结合自动选通, 确保可靠性要求。同时, 通过调整和优化 $\mathrm{GaAs}$ 光阴极发射层厚度, 包括 $\mathrm{Al}_{1-{ }_{x}} \mathrm{Ga}_{x} \mathrm{As}$ 缓冲层厚度和 $\mathrm{Al}$ 组份, 及 $\mathrm{Si}_{3} \mathrm{~N}_{4}$ 过渡层厚 度和光性, 不仅改善了短波长响应度甚至可实现蓝延 伸 ${ }^{[11]}$, 如图 5 所示, 光阴极灵敏度得到显著提高。

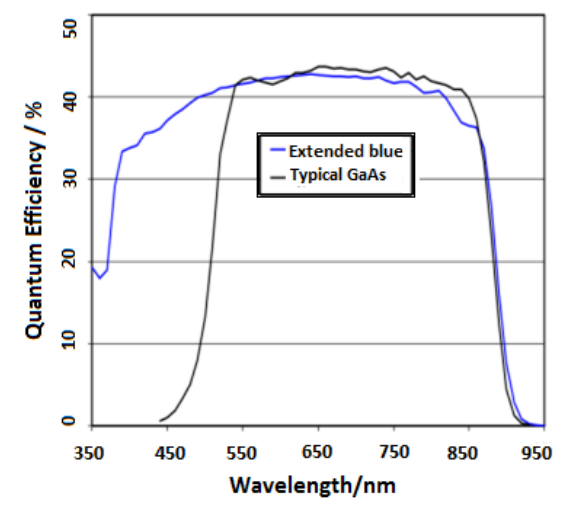

图 5 ITT 的常规和蓝延伸 $\mathrm{GaAs}$ 光阴极的量子效率

Fig. 5 QE curves of typical and extended blue GaAs photocathode

ITT 迅速推出命名为 Pinnacle 的薄膜管, 光阴 极灵敏度最高达到 $2000 \mu \mathrm{A} / \mathrm{lm}$, MCP 噪声因子降 低到 1.7 , 信噪比达到 28 , 分辨力 $64 \mathrm{~lm} / \mathrm{mm}$ 。薄膜 改善了电子透射特性, 还为缩减光阴极和 $\mathrm{MCP}$ 的 间距和场强提供了可能。薄膜管的 MCP 前近贴电 压降低到 $700 \mathrm{~V}$, halo 减小到 $0.90 \mathrm{~mm}^{[12]}$ 。Pinnacle
满足 Omni IV 无膜四代指标要求。2001 年 NVESD 取消 “四代” 的命名而改称为新一代无膜三代, 并 宣布无限期中止 “四代” 开发计划。同时充分肯定 Pinnacle 的技术进步并命名为薄膜三代。ITT 获得 次年的 Omni VI 的绝大部份份额, 其独占最大市场 份额的技术领先地位得到进一步巩固和加强。

Litton 于 2001 年由 Northrop Grumman 收购, 次年又转入 L3, 产品回归薄膜三代, 并通过优化离 子阻挡膜的 $\mathrm{Al}: \mathrm{O}$ 构成比, 来改善膜层在特定电压 的电子透射特性 ${ }^{[13]}$, 以弥补其光阴极技术相比于 ITT 的劣势, 但产品性能始终与 ITT 存在差距。而 无膜三代在 L3 作为一个商业产品得到保留, 最终 达到适度可靠性要求, 在特种部队得到少量装备。

2004 年 DEP 推出自动选通 XR5, 光阴极灵敏度 提高到 $800 \mu \mathrm{A} / \mathrm{lm}$, 信噪比 28 , 分辨力 $64 \mathrm{~lm} / \mathrm{mm}$ 。 超二代管远比三代管要低的前聚焦电压, 可实现更 高速的选通, 可在 $200 \mathrm{~lx}$ 照度级别分辨力下降不超 过 $20 \%$, 亮度增益保持在 $10000 \sim 18000 \mathrm{~cd} \cdot \mathrm{m}^{-2} \cdot \mathrm{x}^{-1}$, 并且 XR5 包括 XD-4 的可靠性都提高到了 $15000 \mathrm{~h}$ 。

2006 年 Photonis 并购 DEP 后将产品整合为 XR-5, XD-4 和 SuperGen。ITT 于 2011 年将夜视业 务剥离为 Exelis 分离上市, 次年美军夜视装备也宣 告全面完成, ITT 开始面向全球市场拓展。此间俄 罗斯 Katod 和德国 Harder Digital 都先后推出了自己 的三代管, 并作为主打产品。但无论是三代, 还是 超二代, 不同厂家产品的性能参数存在相应差别, 即便同一厂家同一生产批次制造的产品, 性能指标 参数都存在一定程度的参差不齐, 所谓的产品系列 实质上大多为同一生产线产品的性能分级, 这也是 像增强器性能参数只给出范围而非确切数值的缘 由, 迭代性能的划定界线变得越来越模糊不清。

像增强器进入高速成长的成熟期。超二代像增 强器已成为与三代像增强器长期并行的技术, 军用 超二代和三代夜视装备已成为一个全球化的市场。

NVESD 于 2013 年对 10 只薄膜三代管和 4 只 XR5 进行了对比测试, 为避免样品由制造商事先选定, 薄膜三代从近 5 年美国军购产品中抽取, 但 Photonis 产品只能通过商业渠道来采购, NVESD 也 承认这并不符合该工作的初衷。结果为两者的分辨 力和 MTF 相差不大, 但前者具有更低的 EBI, 更 高的亮度增益, 饱和度和信噪比, 后者具有更高的 强光分辨力和更小的 halo $^{[2]}$ 。

此间市场上还出现了一种 $16 \mathrm{~mm}$ 管型, 性能与 $18 \mathrm{~mm}$ 管相近, 可满足减小尺寸和重量要求, 各个 厂家也都能生产。还有一种由 Photonis 最早引入的 P45 苂光粉的黑白屏, P45 的光输出覆盖紫色到红 
色，虽然发光效率略低，但在 $380 \sim 500 \mathrm{~nm}$ 集中了 大部分光能, 可利用人眼视觉对黑白图像的处理效 率, 同时保持视杆细胞对蓝绿光的对比度灵敏性。 另外还出现了一种非制冷微测辐射热计的红外焦 平面探测器与像增强器光学集成的微光与热成像 图像融合的增强夜视镜。

自动选通是像增强器的又一个里程碑标志, 薄 膜三代和 XR-5 是三代和超二代各自性能的一次提 升, 并代表各自的最高技术水平。理论上说环境照 度越暗, 观察距离越远, 系统越需要更高的信噪比 和近红外灵敏度以及亮度增益, 以呈现更具良好对 比度的清晰图像。但目前夜间战场环境发生了巨大 变化, 人工照明产生的大气光普遍存在, 在大多数 的场合两者的表现并不会有明显差距。但在固定翼 /直升机的飞行员夜视成像系统 (Aviator's Night Vision Imaging System, ANVIS) 中, 需要滤除蓝绿 光以与机舱照明系统兼容 ${ }^{[14]}$, 三代管的 GaAs 光阴 极的光谱响应特性在这里更具优势, 并且三代管采 用 P43 苂光粉, 而超二代管多采用 P20/P22 苂光粉, 而前者具有更快的衰减时间, 可最小化场景快速变 化产生的运动模糊。

\section{3 超二代的 4G 突破与三代的对策}

在极度黑暗的阴云星光夜晚, 特别是地形反射 极低的山地峡谷, 即使现有最高性能的像增强器都 表现出严重不足, 影响到直升机夜间飞行安全, 因 而对像增强器信噪比和近红外响应提出更高要求。

$\mathrm{GaAs}$ 光阴极灵敏度接近甚至突破 $2500 \mu \mathrm{A} / \mathrm{lm}$, 但近红外响应拓展受限于 GaAs 带隙, 而一种在 GaAs 祄底上外延生长 InGaAs 制作的 NEA 光电阴 极, 截止波长从 $920 \mathrm{~nm}$ 拓展至 $1100 \mathrm{~nm}$, 实现了 1.06 $\mu \mathrm{m}$ 激光的可视化, 但量子效率仍处于较低级别。

$\mathrm{S}-25$ 光阴极灵敏度也已接近甚至突破 $1000 \mu \mathrm{A} / \mathrm{lm}$, 但受限于电子逃逸深度小于光子吸收长度, 进一 步拓展近红外响应, 必须增加吸收厚度, 这将导 致光电子逸出深度过大, 从而无法得到更好的发 射效率。

新技术的进步为现存技术提供了优化改进途 径。借助于微纳制造技术, 来优化光电阴极基底表 面形貌, 增加光子吸收长度且不增加电子逃逸深度, 同时调整介电常数优化近表面电场, 可进一步提高 多碱光电阴极的发射效率。Photonis 借助在光阴极输 入窗内表面制作复杂微结构, 形成一个类似衍射光 栅的功效的光阴极基底, 使投影在输入窗上的入射 光子, 通过这个微结构后将以一个实质性的扩展 角进入光阴极, 从而增大了光子吸收路径, 且不 改变光电子逸出深度, 实现一个可上超 $1000 \mathrm{~nm}$ 下
探 $400 \mathrm{~nm}$ 的拓展光谱范围的多碱光电阴极。结合通 道结构及内壁膜层优化的 MCP, 达到高灵敏度、低 噪声、高增益和高分辨力功效, 辅以超高速自动选 通, 响应时间达到 $0.12 \mathrm{~s}$, 选通开关上升时间达到 $0.5 \mathrm{~S}$ 。Photonis 于 2014 年推出的 $4 \mathrm{G}$ 像增强器, 同 时还提出 $4 \mathrm{G}$ 的技术标准: 光谱响应范围 400 $1000 \mathrm{~nm}$; FOM 大于 1800 ; 分辨力总是高于 57 $1 \mathrm{p} / \mathrm{mm}$, 即使最严重的光污染环境; halo 尺寸不大 于 $0.7 \mathrm{~mm}^{[15]}$ 。

Photonis 产品系列也升级为 $4 \mathrm{G}, \mathrm{XR}-5$ 和 XD-4, 如表 1 所示 ${ }^{[15]}$, $4 \mathrm{G}$ 采用 P43 苂光屏标配, P45 苂 光屏选配, 并且亮度增益约有提高, 信噪比最高接 近 35, 与最高性能的无膜三代管相当, 并且标准测 试还不能充分显示拓展光谱响应的多碱光阴极的 优势, Photonis 宣称, 相比于目前市场上的像增强 器, $4 \mathrm{G}$ 像增强器的探测识别和确认距离提高了 $40 \%$ 。但可能出于避免与 $\mathrm{GaAs}$ 光阴极灵敏度对比 带来误导, 参数不再标示光阴极灵敏度。但 XR-5 和 XD-4 的 MTTF 现在都为 $10000 \mathrm{~h}$, 不知是否相应 地提高了失效阈值。

ITT Exelis 于 2015 年由 Harris 收购。面对 Photonis $4 \mathrm{G}$ 像增强器的突破, 发布了三代优势的 白皮书 ${ }^{[3]}$, 强调三代像增强器的亮度增益和 $\mathrm{EBI}$ 特别是可靠性的优势, 声称按美军标加速可靠性 试验的对比测试结果表明 Photonis 的像增强器仅 有美国三代管生命周期的 $1 / 4$ 甚至 1/5。同时强调 $\mathrm{GaAs}$ 光阴极近红外响应拓展的潜力, 提出通过借 助 InGaAs 并调整 In 组成, 可获得介于 InGaAs 与 GaAs 光阴极之间的量子效率, 如图 6 所示, 同时 得到比拓展光谱响应的多碱光阴极更好的近红外 响应拓展 ${ }^{[3]}$ 。

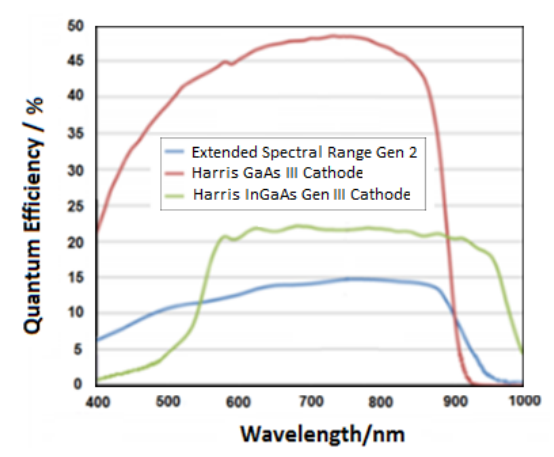

图 6 Harris 的 $\mathrm{GaAs}$ 和 $\mathrm{InGaAs}$ 光电阴极与 Photonis 的 拓展光谱响应的 S-25 光电阴极的量子效率

Fig.6 QE curve of extended spectral range S25 form Photonis, GaAs and InGaAs photocathodes from Harris

2018 年 Harris 推出升级产品 $\mathrm{M} 22 \mathrm{H} / \mathrm{M} 24 \mathrm{H}$, $\mathrm{M} 22 \mathrm{H}$ 信噪比突破了 31 , 而 $\mathrm{M} 24 \mathrm{H}$ 更是提升到 35, 
如表 2 所示, 并采用极限分辨力下的信噪比的标 示形式 ${ }^{[16]}$, 但近红外响应拓展没有实质性进展, 也无法确定离子阻挡膜膜层是否进一步减薄。同 时 Harris 将全系产品按 FOM 值分级, 并将标配 P45 荧光屏的产品新增为一个产品系列。P45 苂光 粉的白屏正发展成为一种趋势, 并已通过美军航 空认证。白中泛绿的黑白图像或将替代绿色图像 成为夜视图像的特征。另外, 虽然离子阻挡膜对 三代像增强器可靠性的作用不容忽视, 但 GaAs 光阴极如不能在实现近红外响应拓展的同时保持 足够高的灵敏度, 无膜 MCP GaAs 光阴极像增强 器将有望成为一个新的装备类别。

2019 年 L3 与 Harris 合并后将两家公司夜视业 务合并, 并出售给以色列的 Elbit System 美国公司。

在各种军用头盔及武器观瞄系统中集成夜视 能力是夜视技术为之持续努力的目标。数字化可集 成的夜视技术是未来的发展趋势, 多种数字夜视的
解决方案已开始崭露头角, 面对可见光/近红外及最 新的短波红外图像传感器等众多技术的竞争, 像增 强器可满足于无月星光微弱照度下的快速成像, 以 及紧凑、轻便和低功耗的可穿戴装备集成能力, 并 未显露可被其他技术所能超越和替代的迹象, 结合 CMOS 读出的 ICMOS 和 EBCMOS 的数字像增强相 机也已成为像增强技术的一种新的产品形态。

\section{2 像增强器的评价方法与标准更新}

夜视装备是实现夜战优势和安全的关键。夜视 仪的性能取决于其夜间目标的探测、识别和确认的 距离, 影响作用距离的外部因素为环境照度及光谱 分布, 目标大小及反射特性, 景物的对比度和动态 范围, 以及背景特性和大气传播特性等, 然后是光 学物镜, 像增强器图像质量和目镜, 以及观察者的 人眼对比度阈值。

表 1 Photonis 像增强器技术参数

Table 1 Main parameters of image intensifier tube of Photonis

\begin{tabular}{|c|c|c|c|c|c|c|c|c|}
\hline & \multicolumn{2}{|c|}{$\mathrm{XD}-4$} & \multicolumn{2}{|c|}{ XR5 } & \multicolumn{2}{|c|}{$4 \mathrm{G}$} & \multicolumn{2}{|c|}{$4 \mathrm{G}+$} \\
\hline & Min. & Typ. & Min. & Typ. & Min. & Typ. & Min. & Typ. \\
\hline FOM (SNR $\times$ resolution $)$ & 1200 & 1400 & 1600 & 1770 & 1800 & 2200 & 2300 & - \\
\hline Signal-to-noise ratio & 20 & 22 & 25 & 26 & 28 & 30 & 30 & 32 \\
\hline Limiting resolution/(lp/mm) & 60 & 64 & 64 & 68 & 64 & 72 & 72 & 76 \\
\hline High-light resolution@200 1x, (min),lp/mm & \multicolumn{2}{|c|}{50} & \multicolumn{2}{|c|}{55} & \multicolumn{2}{|c|}{55} & \multicolumn{2}{|c|}{55} \\
\hline Halo(with $0.35 \mathrm{~mm}$ input spot) $/ \mathrm{mm}$ & \multicolumn{2}{|c|}{0.8} & \multicolumn{2}{|c|}{0.8} & \multicolumn{2}{|c|}{0.7} & \multicolumn{2}{|c|}{0.7} \\
\hline Luminous gain, (min.-max.) @ $2 \times 10^{-5} 1 \mathrm{x}, \mathrm{cd} / \mathrm{m}^{2} / 1 \mathrm{x}$ & \multicolumn{2}{|c|}{$10000-18000$} & \multicolumn{2}{|c|}{$10000-18000$} & \multicolumn{2}{|c|}{$10000-20000$} & \multicolumn{2}{|c|}{$10000-20000$} \\
\hline Max. output brightness, @10 and 2001x, cd $/ \mathrm{m}^{2}$ & \multicolumn{2}{|c|}{$4-17$} & \multicolumn{2}{|c|}{$4-17$} & \multicolumn{2}{|c|}{$4-17$} & \multicolumn{2}{|c|}{$4-17$} \\
\hline $\mathrm{EBI} / \mu \mathrm{lx}$ & \multicolumn{2}{|c|}{$0.15-0.25$} & \multicolumn{2}{|c|}{$0.15-0.25$} & \multicolumn{2}{|c|}{$0.15-0.25$} & \multicolumn{2}{|c|}{$0.15-0.25$} \\
\hline
\end{tabular}

表 2 HARRIS 的 F9400/F9800 系列三代像增强器技术参数

Table 2 HARRIS F9400/F9800 M22H/M24H Gen 3 image intensifier tube

\begin{tabular}{|c|c|c|c|c|c|c|}
\hline & & $\mathrm{M} 16 \mathrm{H}$ & $\mathrm{M} 18 \mathrm{H}$ & $\mathrm{M} 20 \mathrm{H}$ & M22H & $\mathrm{M} 24 \mathrm{H}$ \\
\hline \multicolumn{2}{|l|}{ FOM (maximum) } & 1600 & 1800 & 2000 & 2200 & 2400 \\
\hline \multirow{3}{*}{ Signal-to-noiseratio } & @ 81 lp/mm & - & - & - & $24.7-27.1$ & $27.2-29.6$ \\
\hline & @ 72 lp/mm & - & $22.2-25.0$ & $25.0-27.7$ & $27.8-30.5$ & $30.6-33.3$ \\
\hline & (a) 64 lp/mm & $21.8-25.0$ & $25.0-28.1$ & $28.1-31.2$ & $31.2-34.3$ & $34.4-37.5$ \\
\hline \multicolumn{2}{|c|}{ Resolution, (min.), lp/mm } & 64 & 64 & 64 & 64 & 64 \\
\hline \multicolumn{2}{|c|}{ High-light resolution,@200 lx,(min.),lp/mm } & 36 & 36 & 40 & 40 & 40 \\
\hline \multirow{2}{*}{$\begin{array}{l}\text { Photocathode } \\
\text { sensitivity(min.) }\end{array}$} & @ $2856 \mathrm{~K}, \mu \mathrm{A} / \mathrm{lm}$ & 1800 & 1800 & 2000 & 2200 & 2200 \\
\hline & @ 880 nm, mA/W & 80 & 90 & 100 & 120 & 120 \\
\hline \multirow{2}{*}{$\begin{array}{l}\text { Luminous gain, } \\
\text { (min.-max.), } \mathrm{cd} / \mathrm{m}^{2} / 1 \mathrm{x}\end{array}$} & @ $2 \times 10^{-5} 1 \mathrm{x}$ & $\begin{array}{l}50000 / \pi- \\
80000 / \pi\end{array}$ & $\begin{array}{l}50000 / \pi- \\
80000 / \pi\end{array}$ & $\begin{array}{l}50000 / \pi- \\
80000 / \pi\end{array}$ & $\begin{array}{l}50000 / \pi- \\
80000 / \pi\end{array}$ & $\begin{array}{l}50000 / \pi- \\
80000 / \pi\end{array}$ \\
\hline & $@ 2 \times 10^{-3} 1 \mathrm{x}$ & $\begin{array}{c}14000 / \pi-21000 / \\
\pi\end{array}$ & $\begin{array}{c}14000 / \pi-21000 \\
/ \pi\end{array}$ & $\begin{array}{c}14000 / \pi-2100 \\
0 / \pi\end{array}$ & $\begin{array}{c}14000 / \pi-21000 / \\
\pi\end{array}$ & $\begin{array}{c}14000 / \pi-21000 / \\
\pi\end{array}$ \\
\hline \multicolumn{2}{|c|}{ Out brightness,@10and 2001x,cd/m² } & $9.6-13.7$ & $9.6-13.7$ & $9.6-13.7$ & $9.6-13.7$ & $9.6-13.7$ \\
\hline \multicolumn{2}{|l|}{$\mathrm{EBI}, 1 \mu \mathrm{lx}$} & 2.5 & 2.5 & 2.5 & 2.5 & 2.5 \\
\hline
\end{tabular}


噪声和模糊是影响图像质量的根本因素。微弱 光级别的光子噪声服从泊松分布, 增强图像按其增 益也放大现有光子噪声, 放大传递过程的统计分布 及相关信号损失, 还合并成为另一个额外的噪声分 量。EBI 是与噪声本底相当的环境照度, 室温测量 值约相当阴云星光照度的 $1 / 20$, 在绝大多数使用情 形其影响都微不足道, 除非是高温酷暑阴云星光的 夜晚。随着照度的增加, 图像亮度随之达到满足人 眼光适应状态的亮视觉级别, 图像的细节分辨也逐 渐显现。信噪比可比拟像增强器在微弱光级别得到 的图像质量。人眼最低可接受的图像质量相应的信 噪比的值为 10 。随环境照度增加, 信噪比超过 40, 人眼就不能再察觉出图像信噪比的改善。MTF 成为 评价增强图像质量的最全面指标, 可量化图像对不 同空间频率的分辨能力。像增强器的近贴聚焦和 $\mathrm{MCP}$ 通道间距是增强图像 MTF 高频段的主要限 定, 来自光阴极的光电子在 $\mathrm{MCP}$ 上的反弹和散射 是 MTF 低频段的主要影响因素。随着环境照度的 进一步增加, 离子噪声对 MTF 的全面影响也随着 荧光屏输出亮度逐渐增加而显现, 当亮度增加到设 定阈值, 选通电源自动启动, 亮度达到饱和。

评价像增强器性能的最佳方法是场试。人眼对 图像质量评价最准确, 像增强器的图像质量本身就 是人眼的视觉感受。但毕竟这是一种主观的方法, 个体间也存在差异。系统终端用户需要预期现场使 用特性的定量表征。目前像增强器的性能预估大多 参照制造商提供的按军标规范测试的性能参数。

在军标规范中, 光阴极灵敏度, $\mathrm{EBI}$, 亮度增 益, 饱和度, 极限分辨率, 信噪比, 和固定图案噪 声、亮度均匀性, 斑点等图像疪病, 列为 A 组检验, 要求全数检测, MTF, halo, 强光分辨力, 和亮光 源保护及时间依存特性等, 列为 $\mathrm{B}$ 组检验, 以抽样 形式检测。振动、机械冲击, 环境温度和可靠性则 为 $\mathrm{C}$ 组和 $\mathrm{D}$ 组。像增强器的标准规范源于当年针 对二代管制定的美军标, 但一些测试及指标随着像 增强器升级而进行了相应的完善, 如光阴极灵敏度 除测量白光灵敏度外, 还要测量近红外波段的辐射 灵敏度, 通常二代管在 $800 \mathrm{~nm}$ 和 $850 \mathrm{~nm}$, 三代管 在 $830 \mathrm{~nm}$ 和 $880 \mathrm{~nm}$ 。

在军标规范中, 信噪比测试采用一个 $0.2 \mathrm{~mm}$ 直径的输入光点, 以一个 $10 \mathrm{~Hz}$ 的噪声等效带宽, 测量其在苂光屏上的平均亮度 (信号) 与亮度标准 偏差 (噪声) 之比。允许测量孔径可略大于 $0.2 \mathrm{~mm}$ 。 输入条件为 $2856 \mathrm{~K} \pm 50 \mathrm{~K}$ 的 $10^{-4} 1 \mathrm{x}$ 照度 ${ }^{[17]}$, 相应 于清晰星光的地面照度。信噪比是一个通常仅针对 于光阴极和 $\mathrm{MCP}$ 的质量因数, 最后需通过校正因
子 $K$ 来去除荧光屏的影响, 通常 P-43 的 $K$ 因子为 $1.15, \mathrm{P}-22$ 则为 $1.21^{[2]}$ 。

信噪比测试远非是一个可严格比拟实际应用 特性的测量。照度源于人眼对可见光亮度的感受, 从光度计量上理解这只是一个针对可见光辐射的 测量。 $2856 \mathrm{~K}$ 铇丝白炽灯发射 $300 \sim 2500 \mathrm{~nm}$ 波长 范围的连续光谱, 但近红外辐射实际弱于星光, 如 图 7 所示, 且其光功率和光谱分布随时间而变化, 标准测量并不能充分体现拓展光谱响应光阴极的 优势。并且测量与空间频率几乎没有任何关联。而 从可允许的测量孔径约大于输入光点尺寸来看, 这 里考虑更多的是收集更多的信号，而没有考虑空间 发生改变的信号实质上等效于噪声。Harris 最新采 用的极限分辨力对应的信噪比的标示方法说明准 确的信噪比测量本身就是 FOM, 不过尚未见其测 试方法, 无法对测量准确性进行评判。

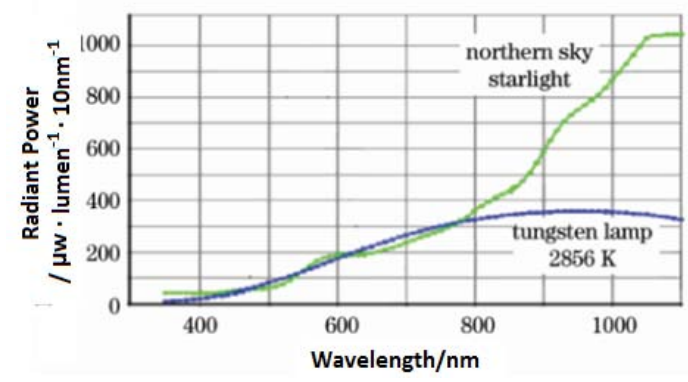

图 7 星光和 $2856 \mathrm{~K}$ 铇丝白炽灯的光谱分布

Fig.7 Spectral distribution of star light and tungsten lamp

MTF 的测量也没有得到很好利用。在军标规范 中, 通过测量最佳照度的狭缝像空间亮度分布, 求 取狭缝像一维线扩散函数的傅里叶变换模, 以不大 于 $0.2 \mathrm{lp} / \mathrm{mm}$ 的零频率幅值归一化处理得到 $\mathrm{MTF}^{[17]}$ 。MTF 的精确测量还可很好地评估极限分 辨力, 但受到眩光遮蔽, 线性度、固定模式噪声和 halo 及空间不变区域等诸多因素影响 ${ }^{[18]}$, 即便仅对 几个离散空间频率有规定 MTF 要求, 测量的准确 性和一致性都普遍存在问题。由此极限分辨力成为 评估像增强器图像清晰度的唯一指标。但其毕竟仅 是反映分辨极限, 与成像清晰度并无必然联系。人 眼在低频部分具有较高分辨能力, 极限分辨力高图 像反而不清晰的事例并不少。而军标中的极限分辨 力测量, 是通过 10 倍放大镜目视观察 USAF 1951 靶标, 由在最佳照度目视可辨明的最高空间频率图 案来量化, 这是一种相当主观的方法。

像增强器的噪声强度很大程度上取决于场景 亮度, 而适度的亮度增益成为一个广泛采用的折衷 取舍。受离子阻挡膜的保护, 总体上三代像增强器 具有更高的亮度增益和饱和度。但离子阻挡膜也非 
离子噪声的绝对屏障, 特别是对于薄膜管, 膜厚成 为信噪比与可靠性之间的一个折衰选择。可靠性测 试可用来评判像增强器对恶劣条件如极端光应力 及曝光时间和高温的承受能力。美军标采用加速可 靠性试验, 光应力较正常试验提高 5 倍, 试验的每 单次循环周期的时间缩短了 5 倍, 但期间的强脉冲 时间占比提高了 5 倍。规定 MTTF 目标值 $10000 \mathrm{~h}$ 的累积加速试验时间为 $2000 \mathrm{~h}$, 最小值 $7500 \mathrm{~h}$ 的累 积加速试验时间 $1500 \mathrm{~h}$ 。超二代管可靠性试验中的 强脉冲强度也增加了 5 倍, 加速试验也非美军标独 家采用。但美军标规定的三代管信噪比失效阈值为 最低不低于 16 , 甚至还按性能等级来划分失效阈 值, 如信噪比起始额定目标值 28.0 最小值 25.0 , 失 效阈值分别为 23.0 和 $21.0^{[19]}$ 。而大多数超二代管 仍在执行信噪比最低不低于 11 , 或起始额定值减半 的失效标准。

FOM 难以简单概括像增强器性能, 军标规范的 全面测试参数同样存在严重不足。标准规范更多的 意义在于产品生产研制过程的质量一致性控制, 而 非特征性能的准确表征。这些参数大多在特定条件 测量, 并不能准确反映实际使用特性。即便是在标 准计量条件的准确测量, 这些参数的组合对不同环 境条件的图像质量影响也远非直观明瞭, 仅能提供 一个粗略的预估。

像增强器的迭代性能体现在黑夜里看的更远 和更清晰的能力, 不仅要在最黑暗的时刻, 同样还 要在有严重光污染的情形。而最新技术的迭代性能 更多的来自于光谱范围拓展和高速自动选通。

Photonis 也提出 $400 \sim 1000 \mathrm{~nm}$ 的拓展光谱和超快自 动选通的 $4 \mathrm{G}$ 技术标准。但以照度为标准的光度计 量, 不能充分体现近红外响应拓展带来的性能优 势。DEP 早年也提出光谱信噪比的概念, 可一直缺 乏相应的标准和测试方法。但即便是采用单色光, 对像增强器近红外响应特性的表征和测量都非常 有必要。同样对高速选通性能的评估也仅仅停留在 强光分辨力, 或简单的选通电源响应时间和上升时 间的测量, 同样远非是一个可严格比拟实际应用特 性的测量。像增强器的标准更新与性能升级同样的 重要而紧迫。而性能参数的精确表征和测量对像增 强器的迭代性能升级同样也有着重要的促进作用。 极限分辨力对应的信噪比测量, 特定照度的 MTF 的准确测试, 都需要加以解决并规范。

夜视装备是实现夜战优势和安全的关键, 开展 像增强器特征参数与实际使用性能对应关系的研 究同样必要而重要。目前大多数制造商都建立有 实验室模拟现场试验的设施装置和测试设备, 并
耗费大量的资源来校准和维护，但普遍缺乏严格 和统一的测试规范和标准, 且同样存在以照度作 为光度计量带来的不足，这些工作没有得到持续 有效的开展 ${ }^{[20-21]}$ 。在可标定且受控条件, 模拟不同 夜间环境的光度和光谱分布, 通过光学透镜耦合的 ICMOS 形式, 结合图像采集及相应的软件处理系 统, 获得和分析像增强器或夜视仪的增强图像, 来 测量像增强器的所有性能指标, 比较增强图像的质 量, 建立人眼视觉与图像质量间的评价关系, 减少 主观观察标准的影响, 并便于对不同像增强器、系 统、配置的直接对比。同时在像增强器的使用过程, 也应建立严格的等级划分及定期检测制度, 并配置 便携式的场试测试仪，以便能够及时对性能退化的 像增强器进行翻新和更换, 确保在确定使用时间段 内保持确定的性能要求。

\section{3 结束语}

随着多碱光阴极的光谱范围拓展取得实质性 突破, 同时无膜 MCP 在一些 GaAs 光阴极像增强器 中也成为可能, 像增强器的 “代” 也彻底失去了意 义, 超二代像增强器已发展成为一种与三代像增强 器并行的技术。同时, 数字像增强相机也已成为像 增强技术的一种新的产品形态。

像增强器的性能体现在黑夜里看的更远和更 清晰的能力, 不仅是最黑暗的时刻, 同样还有严重 光污染的情形。像增强器的最新迭代性能进步更多 的来自于光谱范围拓展和高速自动选通。但 FOM 难以简单概括像增强器性能, 军标规范规定的全面 测试参数同样存在严重不足, 无法充分体现性能升 级带来的迭代性能突破。迭代性能划定和命名之争 贯穿现代像增强器的发展历程, 却始终缺乏一个全 面而准确的对比评判。评价像增强器性能的最佳方 法是场视, 但性能参数的精确表征和测量对像增强 器生产和研制及其性能升级都有着重要的作用, 像 增强器的标准更新及其性能参数与实际使用性能 对应关系的研究已经很有必要, 包括数字夜视的解 决方案及其相应标准。

\section{参考文献：}

[1] Bosch L A. Image intensifier tube performance is what matters[C]//Proc. of SPIE, 2000, 4128: 65-78.

[2] Bender E, Wood M, Hosek D, et al. Characterization of domestic and foreign image intensifier tubes[C]//Proc. SPIE, 2013, 8706: 870607.

[3] Eric Garris. The Gen 3 Advantages[EB/OL].[2018-12-11] https: //www.HARRIS.com/sites/default/files/the-gen-3-advantage-white- 
paperv3.pdf.

[4] Chrzanowsk K. Review of night vision technology[J]. Opto -Electron, 2013, 21(2): 153-181.

[5] Smith C. Detection of special operations forces using night vision devices[R]. ORNL/TM-2001/172， 2001: www.osti.gov/bridge/ product. biblio.jsp.

[6] Vastsia M, Stich U, Dunlap D. Night-Sky Radiant Sterance From 450 to 2000 Nanometers[R]. NTIS, 1972.

[7] Bradley A, Kaiser M, Evaluation of Visual Acuity with Gen III Night Vision Goggles. NASA Technical Memorandum108792[R]. https://ntrs.nasa.gov/archive/nasa/casi.ntrs.nasa.gov/19940019501.p df.

[8] Estera J, Saldana M. Gated power supply technologies for advanced image intensifiers[C]//Proc. SPIE, 2003, 4796: 60-70.

[9] Estrera J P, Ford C E, Giordana A, et al. High reliability GaAs image intensifier with unfilmed microchannel plate[C]//Proc. SPIE, 1999, 3749: $713-714$

[10] Estrera J, Bender E, Giordana A, et al. Long lifetime generation IV image intensifiers with unfilmed microchannel plate[C]//Proc. SPIE, 2000, 4128: 46-53.

[11] Smith A, Passmore K, Sillmon R. Transmission mode photocathodes covering the spectral range[C/OL]//New Developments in Photodetection 3rd Beaune Conference, 2002: https://www. researchgate.net/publication/284829644_Transmission_mode_phot ocathodes_covering_the_spectral_range.

[12] Floryan N, Devoe T, Peck T, New image intensifier family for military and homeland defense[C]//Proc. SPIE, 2003, 5071: 397-501.
[13] Sinor T W, Estera J P. An analysis of electron scattering in thin dielectric films used as ion barriers in generation III image tubes[C]//Proc. SPIE, 2003, 4796: 23-32.

[14] MIL-STD-3009. Lighting, Aircraft, Night Vision Imaging System (NVIS) Compatible: Superseding MIL-L-85762A[S/OL]. 2001: https://appliedavionics. com/pdf/ MIL- STD- 3009.pdf.

[15] Photonis Corp. XD-4/XR5/4G/4G+ Technical Informatio [EB/ OL]. [2019-02-06]. https://www.photonis.com/products/image-intensifier-tube-4g.

[16] Harris Corp. $18 \mathrm{~mm}$ Gen3 image intensifier Spec. Sheet [EB/OL]. [2019-02-11]. https://www.harris.com/solution/image-intensifier- tubes.

[17] MIL-STD-1858. Performance Parameters of Image Intensifier Assemblies[S/OL]. DOD, 1981, http://aunv.blackice. com.au/ userfiles/david-mx_10160_milspec_2009_MX-10160-GS_TUBE_ SPEC.pdf.

[18] Ortiz S, Otaduy D, Dorronsoro C. Optimum parameters in image intensifier MTF measurements[C]//Proc. of SPIE, 2004, $\mathbf{5 6 1 2}$ $382-391$.

[19] PS/09/JXQR/078,Performance Specification Image Intensifier Assembly. $18 \mathrm{~mm}$ Microchannel Wafer High Performance Tube MX-10160 GS[S]. http://aunv.blackice.com.au/userfiles/davidmx_10160_milspec_2009_MX-10160-GS_TUBE_SPEC.pdf.

[20] Laurent N, Lejard C, Deltel G, et al. Performance characterization of night vision equipment based on triangle orientation discrimination (TOD) methodology[C]//Proc. SPIE, 2013, 8706: 43-55.

[21] Zacher J, Brandwood T, Thomas P. Effects of image intensifier halo on perceived layout[C]//Proc. of SPIE, 2007, 6557: 65570U. 